\title{
Radio luminosity function of brightest cluster galaxies
}

\author{
Z. S. Yuan ${ }^{1,2}$, J. L. $\operatorname{Han}^{1 \star}$ and Z. L. Wen ${ }^{1}$ \\ ${ }^{1}$ National Astronomical Observatories, Chinese Academy of Sciences, 20A Datun Road, Chaoyang District, Beijing 100012, China \\ ${ }^{2}$ School of Astronomy, University of Chinese Academy of Sciences, Beijing 100049, China
}

Accepted 2016 May 10. Received 2016 May 9; in original form 2016 February 15

\begin{abstract}
By cross-matching the currently largest optical catalog of galaxy clusters and the NVSS radio survey database, we obtain the largest complete sample of brightest cluster galaxies (BCGs) in the redshift range of $0.05<z \leq 0.45$, which have radio emission and redshift information. We confirm that more powerful radio BCGs tend to be these optically very bight galaxies located in more relaxed clusters. We derived the radio luminosity functions of BCGs from the largest complete sample of BCGs, and find that the functions depend on the optical luminosity of BCGs and the dynamical state of galaxy clusters. However, the radio luminosity function does not show significant evolution with redshift.
\end{abstract}

Key words: galaxies: clusters: general — galaxies: luminosity function

\section{INTRODUCTION}

Among many galaxies embedded in hot gas inside a galaxy cluster, the brightest cluster galaxy (BCG) is the most massive and luminous galaxy located near the center of the cluster (e.g. Wen \& Han 2015a). BCGs of galaxy clusters differ from other elliptical galaxies in many aspects because of unique cluster environments they inhabits in and the evolution history they have experienced (e.g. Rafferty et al. 2006; Ma et al. 2013). The nuclei of BCGs host supermassive black holes (e.g. Rafferty et al. 2006), so that BCGs manifest their active nuclei by radio jets which is the feedback to cluster medium (see McNamara \& Nulsen 2007). BCGs are more likely to be radio loud than other galaxies (e.g. Burns et al. 1981; Best et al. 2007). The radio emission of BCGs is related to both nuclei activities of BCGs and cluster properties.

Previously there has been much effort to decouple the effect of galaxy properties and of cluster environment on radio emission of BCGs. Without the large radio sky survey data, small samples of galaxy clusters, usually less than a hundred, have been observed for statistics in radio band (e.g. Burns et al. 1981; Zhao et al. 1989; Burns 1990; Ball et al. 1993). The fraction of BCGs being radio-loud above a given threshold of radio flux density or luminosity has often been investigated for some cluster samples to exam possible links between BCG radio emission and the cluster cooling flows (e.g. Peres et al. 1998; Mittal et al. 2009) or cluster mass and X-ray luminosity (e.g. Lin \& Mohr 2007; Ma et al. 2011; Stott et al. 2012) or dynamic states (e.g. Lin \& Mohr 2007; Kale et al. 2015). Mittal et al. (2009) found that BCG radio luminosities were correlated with cluster cooling time, the mass of supermassive black holes and also X-ray luminosity of strong coolcore clusters. Kale et al. (2015) found that a larger fraction of BCGs in relaxed clusters is radio loud than those in merging clusters.

* E-mail: hj1@nao.cas.cn
Because of large data scatters, the statistics can be improved by using large samples of BCGs observed by radio survey data. von der Linden et al. (2007) and Best et al. (2007) cross-identified 625 BCGs of the C4 cluster sample (Miller et al. 2005) with the radio data of NVSS and FIRST surveys (Condon et al. 1998; Becker et al. 1995), and found that the fraction of radio loud BCGs depends on optical luminosity or the stellar mass of BCGs, but not on the cluster velocity dispersion. Antognini et al. (2012) got a sample of 151 FR-II type BCGs by looking at the NVSS and FIRST images of the MaxBCG clusters (Koester et al. 2007), and concluded similarly that both the jet power and the radio loud fraction is correlated with the $r$-band luminosity of BCGs but not the cluster richness. By cross-matching the MaxBCG cluster catalog (Koester et al. 2007) with the FIRST radio survey data (Becker et al. 1995), Croft et al. (2007) obtained a very large sample of 2,615 radio BCGs with $L_{1.4 \mathrm{GHz}}>10^{23} \mathrm{~W} \mathrm{~Hz}^{-1}$, and confirmed that the radio loud fraction depends on the $r$-band absolute magnitude and hence the converted stellar mass of BCGs, from about $5 \%$ at $10^{10.7} \mathrm{M}_{\odot}$ to about $30 \%$ at $10^{11.6} \mathrm{M}_{\odot}$ and that the fraction is larger for the BCGs in richer clusters. Ma et al. (2013) obtained a large sample of 357 radio BCGs by cross-correlating galaxy clusters in eight X-ray catalogs and NVSS radio sources, and confirmed that the radio fraction increases moderately with redshift and cluster X-ray luminosity. They also found that the radio power is greater in more massive clusters and at higher redshifts, which implies possible redshift evolution of BCG radio emission power. Recently, Hogan et al. (2015) investigated 437 radio BCGs with multi-frequency observational images, and found that the core emission is more frequently associated with BCGs with [O III] emission that is the canonical tracer for AGN activity. See Table 1 for a summary of these previous samples.

More fundamental studies of the BCG radio emission or its difference from other galaxies and their dependence on cluster properties should work on the radio luminosity function, which is 
Table 1. A list of statistical studies of radio BCGs with a sample size over 100.

\begin{tabular}{lccccc}
\hline Authors & Cluster sample & Redshift range & Radio data & Flux limit & No. of radio BCGs \\
\hline Lin \& Mohr (2007) & 342 NORAS/REFLEX & $z<0.2$ & NVSS & $10 \mathrm{mJy}$ & 122 \\
von der Linden et al. (2007) & 625 C4 clusters & $0.02<z<0.1$ & NVSS/FIRST & $5 \mathrm{mJy}$ & 252 \\
Best et al. (2007) & 484 C4 clusters & $0.02<z<0.1$ & NVSS/FIRST & $5 \mathrm{mJy}$ & 252 \\
Croft et al. (2007) & 13,240 MaxBCG & $0.1 \leq z \leq 0.3$ & FIRST & & 2,615 \\
Antognini et al. (2012) & 13,823 MaxBCG & $0.1 \leq z \leq 0.3$ & FIRST/NVSS & $0.75 \mathrm{mJy}$ & 151 \\
Ma et al. (2013) & $685 \mathrm{X}$ clusters & $0.1 \leq z \leq 0.6$ & NVSS & $3 \mathrm{mJy}$ & 357 \\
Hogan et al. (2015) & 720 REFLEX/(e)BCS & $0.03<z \leq 0.45$ & NVSS/SUMSS/ATCA/VLA & $15 \mathrm{mJy}$ & 437 \\
This work & $62,686 \mathrm{WH} 15$ & $0.05<z \leq 0.45$ & NVSS/FIRST & $5 \mathrm{mJy}$ & 7,138 \\
\hline
\end{tabular}

a measure of the variation of BCG space density with radio luminosity. To construct a good radio luminosity function a complete sample of objects is needed with known redshifts as well as good measurements of radio emission. Such studies have often been done for galaxies and AGNs (e.g. Condon 1989; Donoso et al. 2009; Best \& Heckman 2012; Simpson et al. 2012; van Velzen et al. 2012; Mao et al. 2012; Best et al. 2014). The radio luminosity functions for AGNs and star-forming galaxies have been found to be very different (e.g. Sadler et al. 2002; Mauch \& Sadler 2007) but no significant evolution with redshift (e.g. Padovani et al. 2011; McAlpine \& Jarvis 2011; McAlpine et al. 2013; Padovani et al. 2015; Prescott et al. 2016). The radio luminosity functions for BCGs have been tried based on small samples of radio measurements (e.g. Ball et al. 1993; Lin \& Mohr 2007).

In this paper, we cross-match the currently largest optical catalog of galaxy clusters (Wen \& Han 2015b) with the largest radio survey database of the NVSS and FIRST (Condon et al. 1998; Becker et al. 1995). As shown in Section 2, we get the largest complete sample of radio BCGs. With such a sample, we study the possible dependence of BCG radio emission on BCG properties and cluster environment in Section 3, and then work on the radio luminosity function of BCGs in Section 4. The conclusions are given in Section 5.

Throughout this paper, we assume a $\Lambda$ CDM cosmology, taking $H_{0}=100 h \mathrm{~km} \mathrm{~s}^{-1} \mathrm{Mpc}^{-1}$, with $h=0.7, \Omega_{\mathrm{m}}=0.3$ and $\Omega_{\Lambda}=0.7$.

\section{BCG SAMPLE AND RADIO EMISSION POWER}

Based on the photometric data of the Sloan Digital Sky Survey (SDSS) Data Release 8 (DR8, Aihara et al. 2011), Wen et al. (2012) identified 132,684 galaxy clusters. Recently, Wen \& Han (2015b, hereafter WH15) have updated the parameters of these clusters with spectroscopic redshift data in DR12 (Alam et al. 2015) and further identified 25,419 new clusters. In total there are 158,103 galaxy clusters in the WH15 cluster catalog. This sample of galaxy clusters is complete up to redshift $z \sim 0.5$ for massive clusters of $M_{500}>2 \times 10^{14} \mathrm{M}_{\odot}$ (see Figure 6 in Wen et al. 2012), which was further verified by their redshift distribution in Figure 7 of Wen \& Han (2015b). Here we take 62,686 galaxy clusters with a redshift $z \leq 0.45$ and a richness $R_{L *} \geq 12$ in the BOSS DR12 sky region of 9,376 square degrees as the parent sample in the following study. The cluster parameters, such as richness $R_{L *}$ and redshift $z$ (including 56,340 spectroscopic and 6436 photometric redshifts), of these clusters are directly taken from Wen \& Han (2015b). As shown in Wen \& Han (2015b), the cluster richness $R_{L *}$ is a good measure of optical mass proxies with a scatter of
0.17 dex. The $r$-band absolute magnitude of BCGs is corrected for redshift evolution by using $M_{\mathrm{r}}^{\mathrm{e}}=M_{\mathrm{r}}^{\mathrm{SDSS}}+Q z$, with $Q=1.16$ as done in Wen \& Han (2015b). The BCG dominance, defined as the difference of absolute magnitudes of the first and second BCGs, $M_{\mathrm{r}, 2}-M_{\mathrm{r}, 1}$, can statistically indicate the dynamic state of galaxy clusters (Wen \& Han 2013).

To get the radio emission flux densities of these BCGs, we cross-match the optical BCG sample with the NVSS radio source catalog which is $99 \%$ complete above $3.5 \mathrm{mJy}$ (Condon et al. 1998). Our approach is quite similar to those in Best et al. (2005a), and we obtain our radio BCG sample with a few steps. First, the radio flux density limit of $5 \mathrm{mJy}$ is adopted to ensure two individual components of a BCG above $2.5 \mathrm{mJy}$ not being missed, and we find the NVSS radio sources within a projection distance of 500 kpc from BCGs, which in general is sufficiently large to pick up radio emission components from BCGs. 15,387 BCGs are found to be associated with 18,600 radio sources. Naturally some of these sources come from BCGs, but others are background radio sources or the radio emission from other galaxies instead of BCGs. Among them, 12,885 BCGs have only one NVSS source, and 1,985 BCGs have two and 517 BCGs have three or more NVSS sources within the projected $500 \mathrm{kpc}$.

Second, we check the physical association more carefully, and adopt a more restrict criterion for the association which is the projection distance less than $50 \mathrm{kpc}$. For BCGs with only one NVSS source, 5,410 BCGs have one coincident NVSS source within 50 kpc which are all accepted as radio BCGs; 6,526 BCGs have a NVSS source outside $100 \mathrm{kpc}$ from the optical position are directly declined as radio BCGs; 257 of the rest 949 BCGs with one NVSS source between 50 and $100 \mathrm{kpc}$ are accepted as radio BCGs, because either high-resolution FIRST images indicate the physical association or the NVSS source peak is coincident with an optical BCG within $50 \mathrm{kpc}$ (see Figure 1 for example images). For 1,985 BCGs with two NVSS sources, we found that 839 of 1,985 BCGs have one NVSS source within $50 \mathrm{kpc}$ though there is the other source beside with a very different flux density, which we adopt as radio BCGs with a flux of the coincident source; 28 BCGs are coincident with one of the double sources which have a similar flux densities (within a factor of 2) coming from radio lobes or jets of a radio galaxy at the middle position of two sources, and hence are not radio BCGs; 226 BCGs with two NVSS sources outside $50 \mathrm{kpc}$ are adopted as radio BCGs, because the FIRST and/or NVSS images clearly indicate the association of a BCG and double sources. Similarly, for 517 BCGs with three or more than three NVSS sources in $500 \mathrm{kpc}$, we adopt: 1) 275 of them as radio BCGs because one NVSS source is within $50 \mathrm{kpc}$ from a BCG (excluding 5 BCGs coincident with one of double sources); 2) 91 BCGs with 


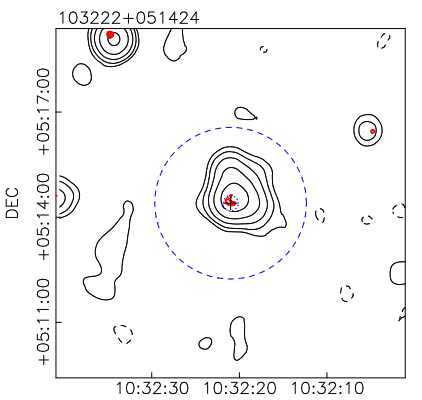

$\mathrm{RA}$

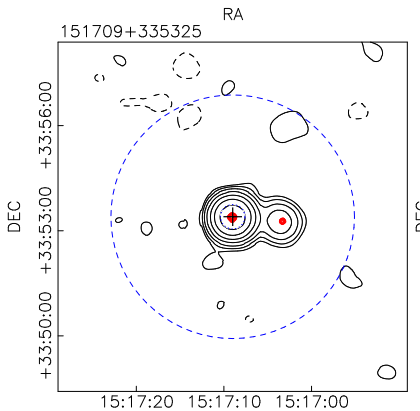

RA

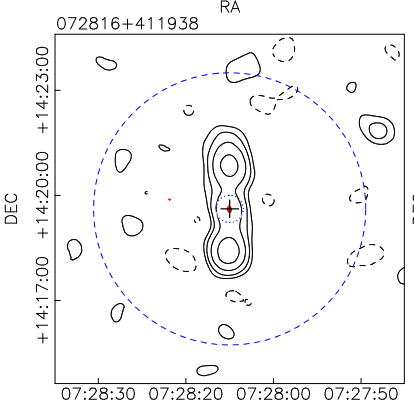

R

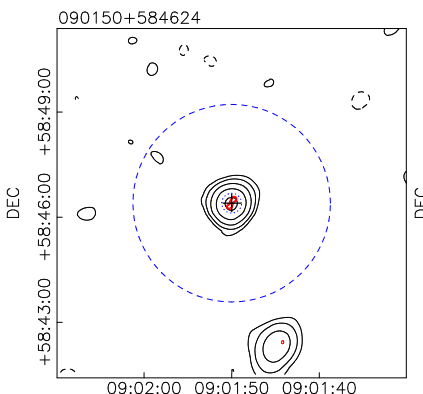

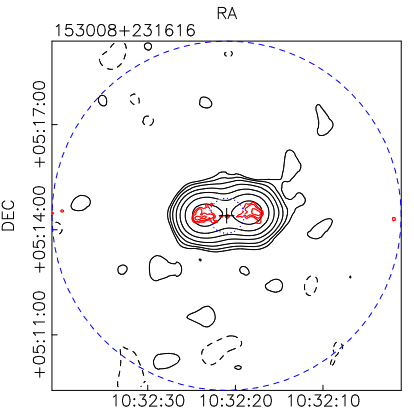

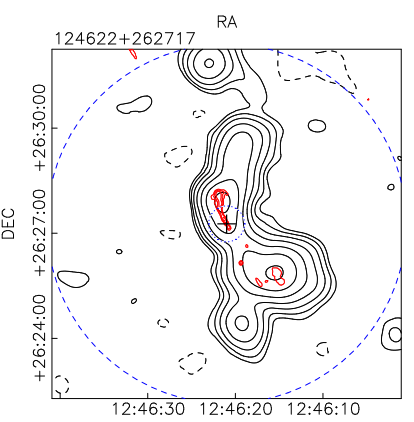

RA
Figure 1. Example images for 6 radio BCGs. Low-resolution (black) contours are plotted for the NVSS data and high-resolution (red) contours for the FIRST data at levels of $\pm 1,2,4, \ldots \mathrm{mJy} /$ beam, with the central cross indicating the optical position of a BCG. The big dashed circle indicates the projection distance of $500 \mathrm{kpc}$ and the small dotted circle near the cross stands for $50 \mathrm{kpc}$ from a BCG. Some radio sources are well coincident with BCGs within $50 \mathrm{kpc}$, and a small number of double or triple sources have the dominant flux from jets or lobes outside $50 \mathrm{kpc}$ from a BCG.

NVSS sources outside $50 \mathrm{kpc}$ are adopted as radio BCGs because the FIRST and/or NVSS image clearly show the association of the BCGs and jets or core. In short, we identified 7,138 radio BCGs in total including 5,667 BCGs with one NVSS source, 1,105 BCGs with two and 366 BCGs with three or more NVSS sources, see a list in Table 2 for the optical and radio parameters. The offset of radio sources from optical BCG positions are shown in Figure 2.

We take flux densities of radio sources from the low resolution survey NVSS to calculate radio emission power of BCGs. Note that the flux densities of identified multiple radio components of a BCG have to be added together to get $S_{1.4 \mathrm{GHz}}$, and then the radio power is obtained through

$P_{1.4 \mathrm{GHz}}=4 \pi D_{\mathrm{L}}^{2} \times S_{1.4 \mathrm{GHz}} \times(1+z)^{1-a}$,

here $P_{1.4 \mathrm{GHz}}$ is in $\mathrm{W} \mathrm{Hz}^{-1}, D_{\mathrm{L}}=(1+$ z) $\frac{c}{H_{0}} \int_{0}^{z} \frac{d z^{\prime}}{\sqrt{\Omega_{m}\left(1+z^{\prime}\right)^{3}+\Omega_{\Lambda}}}$ is the luminosity distance of a cluster at a redshift $z, S_{1.4 \mathrm{GHz}}$ is the radio flux at $1.4 \mathrm{GHz}$ from the NVSS, $(1+z)^{(1-a)}$ is the $k$-correction term with the spectral index $a$ of radio BCGs. We adopt the statistical mean of $a=0.74$

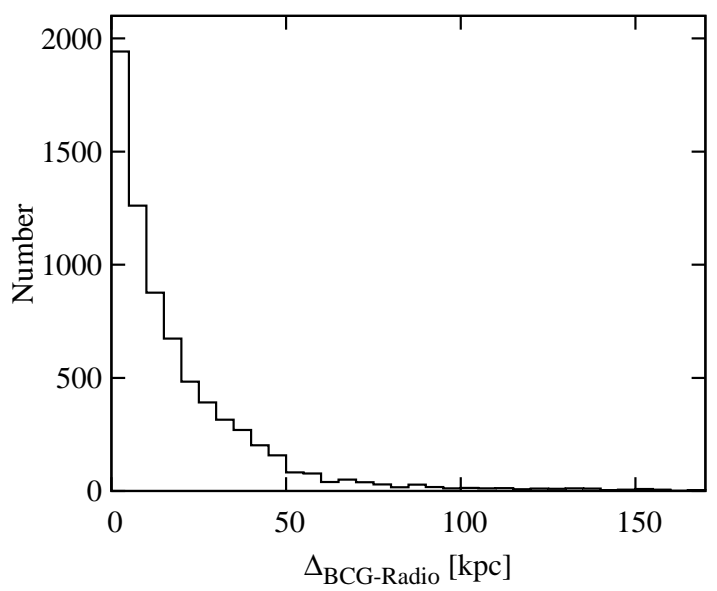

Figure 2. Offsets between optical positions and radio coordinates for 7,138 radio BCGs.

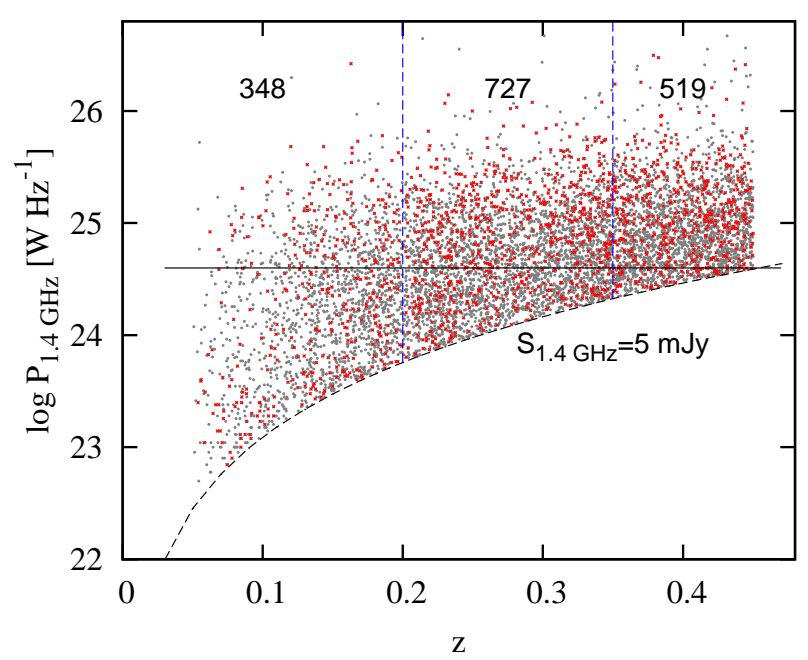

Figure 3. Radio emission power of 7,138 BCGs. All BCGs have a radio flux density $S_{1.4 \mathrm{GHz}}>5 \mathrm{mJy}$. BCGs of a complete sample of 1,594 massive clusters of $M_{500}>2 \times 10^{14} \mathrm{M}_{\odot}$ (equivalent to $R_{L *}>35$ ) are indicated by (red) crosses. Sub-samples for three redshift ranges are divided by vertical dashed lines, which will be used for checking the redshift evolution of the BCG radio luminosity function in Section 4. Above a black line of $\log P_{1.4 \mathrm{GHz}}=24.6$ is a power-limited complete sample of radio BCGs up to $z=0.45$.

obtained by Lin \& Mohr (2007) for all sources. The radio power distribution of 7,138 BCGs is shown in Figure 3.

Among them, 1,594 radio BCGs come from massive clusters of $M_{500}>2 \times 10^{14} \mathrm{M}_{\odot}$, which form a radio-flux-limited complete sample of BCGs that can be used for deriving the radio luminosity function of BCGs in Section 4. We are confident that the massive cluster sample is nearly $100 \%$ complete during the cluster identification (Wen et al. 2012), and the detection of radio emission for $S_{1.4 \mathrm{GHz}}>5 \mathrm{mJy}$ is at least $95 \%$ complete in the low-resolution NVSS survey even when BCGs have two components. On the other hand, the radio detection of BCGs is power-limited complete above a threshold of $\log P_{1.4 \mathrm{GHz}}>24.6$ up to $z=0.45$. 
Table 2. The optical and radio parameters for 7,138 radio BCGs (see online Supporting Information for the full table).

\begin{tabular}{ccccccccc}
\hline $\begin{array}{c}\mathrm{RA} \\
(\mathrm{J} 2000)\end{array}$ & $\begin{array}{c}\mathrm{Dec} \\
(\mathrm{J} 2000)\end{array}$ & $z$ & $\begin{array}{c}R_{L *} \\
(\mathrm{~L} *)\end{array}$ & $\begin{array}{c}M_{\mathrm{r}}^{\mathrm{e}} \\
(\mathrm{mag})\end{array}$ & $\begin{array}{c}M_{\mathrm{r}, 2}-M_{\mathrm{r}, 1} \\
(\mathrm{mag})\end{array}$ & $\begin{array}{c}S_{1.4 \mathrm{GHz}} \\
(\mathrm{mJy})\end{array}$ & $\begin{array}{c}P_{1.4 \mathrm{GHz}} \\
\left(10^{24} \mathrm{~W} \mathrm{~Hz}^{-1}\right)\end{array}$ & $\begin{array}{c}\Delta_{\mathrm{BCG}-\mathrm{Radio}} \\
(\mathrm{kpc})\end{array}$ \\
\hline 0.20327 & 8.66654 & 0.4085 & 31.24 & -22.95 & 0.35 & 11.8 & 7.25 & 11.4 \\
0.20376 & -3.01915 & 0.3732 & 22.17 & -22.78 & 0.66 & 15.6 & 7.69 & 5.7 \\
0.20552 & -0.84525 & 0.4110 & 28.58 & -23.18 & 1.00 & 24.2 & 15.09 & 33.9 \\
0.27341 & 34.46576 & 0.2474 & 23.53 & -23.09 & 1.44 & 37.6 & 7.00 & 14.8 \\
0.31298 & -8.44618 & 0.3288 & 17.69 & -23.01 & 0.43 & 151.1 & 54.95 & 19.0 \\
0.32537 & 28.99514 & 0.4247 & 43.16 & -23.92 & 1.22 & 10.9 & 7.36 & 5.0 \\
0.42376 & 1.98004 & 0.4377 & 29.31 & -23.17 & 0.28 & 5.5 & 4.00 & 69.8 \\
0.47782 & 5.66544 & 0.2399 & 14.48 & -22.89 & 1.01 & 29.3 & 5.08 & 42.5 \\
0.53473 & 19.29001 & 0.1469 & 12.44 & -23.81 & 0.55 & 17.6 & 1.00 & 37.3 \\
0.54585 & 27.82813 & 0.3354 & 27.48 & -23.37 & 1.08 & 5.4 & 2.06 & 16.3 \\
0.56685 & 14.85623 & 0.2967 & 13.02 & -23.10 & 0.74 & 37.6 & 10.71 & 114.6 \\
0.57405 & -7.26382 & 0.3257 & 19.03 & -23.38 & 0.88 & 14.1 & 5.01 & 19.3 \\
0.60304 & -0.54798 & 0.2902 & 27.76 & -23.04 & 0.18 & 19.0 & 5.14 & 24.4 \\
0.67632 & -0.22217 & 0.2989 & 26.52 & -23.36 & 0.82 & 40.8 & 11.83 & 20.5 \\
0.68671 & -1.82153 & 0.3941 & 17.68 & -23.05 & 0.84 & 14.2 & 7.99 & 6.9 \\
\hline
\end{tabular}

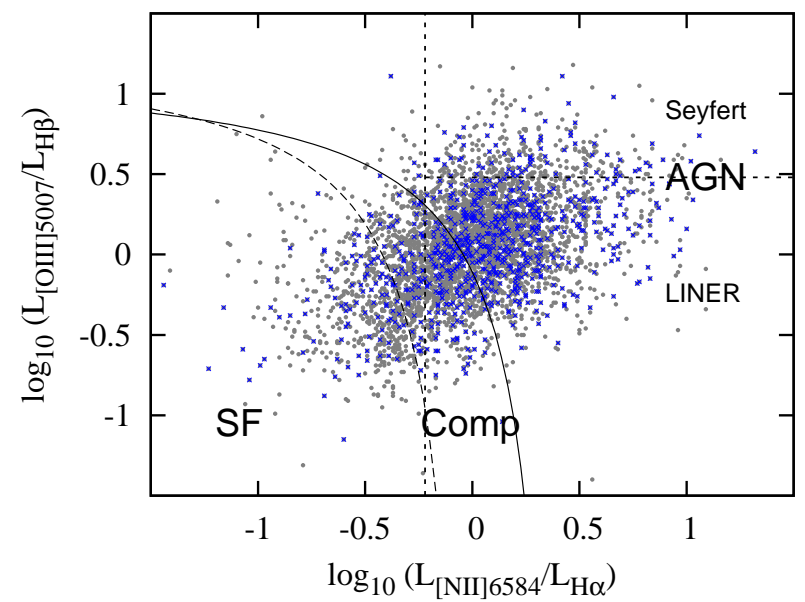

Figure 4. The BPT diagram of emission line ratios for 2,763 BCGs which have a spectrum with a median signal to noise ratio greater than 10 . Among them 619 BCGs have their radio emission detected with $S_{1.4 \mathrm{GHz}}>5 \mathrm{mJy}$, as indicated by little (blue) crosses. The solid curve is the demarcation for defining star-forming (SF) galaxies (Kewley et al. 2001), and the dash curve is the demarcation for classifying AGNs (Kauffmann et al. 2003). Dotted lines on $L_{[\mathrm{OIII}] 5007} / L_{\mathrm{H} \beta}=3$ and $L_{[\mathrm{NII}] 6584} / L_{\mathrm{H} \alpha}=0.6$ are used to dividing LINERs and Seyferts.

\section{FRACTION OF RADIO BCGS AND DEPENDENCE ON BCG AND CLUSTER PROPERTIES}

As shown above, only 7,138 BCGs (11.4\%) of 62,686 galaxy clusters of $z<0.45$ have radio emission with a flux density of $S_{1.4 \mathrm{GHz}}>5 \mathrm{mJy}$. No doubt that more BCGs can be detected in radio via more sensitive observations. In this section we check the possible dependence of the radio fraction on BCG characteristics and cluster properties.

\subsection{Radio fraction of spectroscopically classified BCGs}

Most BCGs are elliptical galaxies with active nuclei, showing AGN properties. Nevertheless a few percent of galaxy clusters show a cooling flow near the center of clusters (see Fabian 1994; Allen et al. 2011), which is probably related to the on-going or post
Table 3. Radio fraction of spectroscopically classified BCGs

\begin{tabular}{lrrrr}
\hline \hline BCG samples & SF & Comp & AGN & sum \\
\hline BCGs with 4 lines detected & 357 & 1103 & 1303 & 2763 \\
No. of Radio BCGs & 67 & 161 & 391 & 619 \\
Radio fraction & $18.8 \%$ & $14.6 \%$ & $30.0 \%$ & $22.4 \%$ \\
BCG No. of log $P_{1.4 \mathrm{GHz}}>24.6$ & 25 & 57 & 151 & 233 \\
Radio fraction & $7.0 \%$ & $5.2 \%$ & $11.6 \%$ & $8.4 \%$ \\
\hline
\end{tabular}

star formation features of BCGs (e.g. O'Dea et al. 2010; Liu et al. 2012a,b). It is intriguing to know how many BCGs have AGNs and how many of them possess star formation features.

In general galaxies can be broadly classified as starforming galaxies, radio-loud AGNs, and composites (e.g., Machalski \& Godlowski 2000; Sadler et al. 2002; Best et al. 2005a; Mauch \& Sadler 2007) according to spectra of galaxies or their nuclei. The BPT diagram of line ratios (Baldwin et al. 1981) has widely been used as a diagnostic to separate radio-loud AGNs from star-forming galaxies (e.g., Kauffmann et al. 2003; Best et al. 2007; Best \& Heckman 2012). In our parent sample of 62,686 galaxy clusters, 56,340 BCGs have their spectra observed already. However, to get the line ratios among $\mathrm{H} \alpha, \mathrm{H} \beta$, [OIII]5007 and [NII]6584, the spectra of BCGs should have a good signal-to-noise ratio. From the value-added spectroscopic catalogs ${ }^{1}$ produced by a research group from the Max Planck Institute for Astrophysics and the Johns Hopkins University (see Tremonti et al. 2004; Brinchmann et al. 2004), 2,763 BCGs have a spectrum with a median signal-to-noise ratio per pixel of the whole spectrum $s n_{\text {median }}>10$ and with the pipeline warning flags $z$ Warning $=0$, from which four spectral lines of $\mathrm{H} \alpha, \mathrm{H} \beta$, [O III]5007 and [N II]6584 are significantly detected. The BPT diagram of 2,763 BCGs is plotted in Figure 4. According to the demarcation for defining star-forming galaxies and AGNs (Kewley et al. 2001; Kauffmann et al. 2003), 1,303 (47.2\% of 2,763) BCGs have a AGN, 357 BCGs (12.9\%) have star-forming features, and the rest 1,103 BCGs are composite galaxies. Among all these BCGs,

${ }^{1}$ http://wwwmpa.mpa-garching.mpg.de/SDSS/DR7/ 
619 (22.4\% of 2,763) BCGs have radio emission detected above $S_{1.4 \mathrm{GHz}}=5 \mathrm{mJy}$, as indicated in Figure 4.

The subsample of BCGs with the four lines consists of bright nearby (majority $z<0.2$ ) BCGs, not a complete sample by any means. Nevertheless, the statistics on the radio fraction of these BCGs given in Table 3 and the BPT diagram in Figure 4 can at least tell that not all radio BCGs possess AGNs, and that $10.8 \%$ (i.e. 67/619) radio BCGs are star-forming galaxies, not necessary just elliptical galaxies. The AGN percentage is larger for radio BCGs (i.e. $63.2 \%=391 / 619$ ) than for BCGs in general (i.e. $47.2 \%=$ 1303/2763).

\subsection{Radio power versus optical characteristics of BCGs}

The BCGs are unusual galaxies. By using the large sample of 7,138 radio $\mathrm{BCGs,}$ we here investigate the possible dependence of $\mathrm{BCG}$ radio power on the absolute magnitude, velocity dispersion, and luminosity of [O III]5007 line. The absolute $r$-band magnitude is an indicator of stellar mass of galaxies and is also related to the mass of a central black hole (e.g. see Eq.(8-10) in Mittal et al. 2009). The stellar velocity dispersion has been well-established to be related to the mass of central black hole (Tremaine et al. 2002; Marconi \& Hunt 2003). While the high ionization [O III] forbidden line is emitted by filamentary gas related to jets (McCarthy 1993), hence it is the indicator of AGN activity (e.g. Maiolino \& Rieke 1995). Beside the evolution corrected $r$-band absolute magnitude of 7,138 radio BCGs, we obtain the stellar velocity dispersion $\sigma$ of 2,884 BCGs and the luminosity of [O III]5007 line of 1,237 BCGs from the value-added spectroscopic catalogs pproduced by a research group from the Max Planck Institute for Astrophysics and the Johns Hopkins University (see Tremonti et al. 2004; Brinchmann et al. 2004).

As shown the top panels of Figure 5, the radio power data in all three panels are rather scattered against the absolute magnitude, the velocity dispersion and [O III]5007 line luminosity of BCGs. Our results are consistent with the plots previously obtained for a small sample of BCGs by Mittal et al. (2009) and large samples of BCGs by Croft et al. (2007), Antognini et al. (2012) and Hogan et al. (2015). The mild or strong correlation between radio power and the absolute magnitude found for radio galaxies (e.g. Calvani et al. 1989) is not shown for radio BCGs, even for the complete sample of radio BCGs in massive clusters. No significant correlation between the radio power and stellar velocity dispersion is found for BCGs. The Spearman rank-order correlation coefficient $r$ in each panel tells how strong the correlation is. Nevertheless, a weak correlation $\left(r^{\prime}=0.41\right)$ exists between the radio power and luminosity of [O III] line of BCGs as shown in the top right panel of Figure 5, though not as strong as for radio galaxies (Saunders et al. 1989; McCarthy 1993; Willott et al. 1999; Sikora et al. 2013). Very probably line emission is fundamentally related with core radio emission (Hogan et al. 2015) of high-excitation radio galaxies (Best \& Heckman 2012). The radio power of BCGs in this paper includes all radio emission components, not just limited to core.

Note that the fraction of radio loudness of BCGs has been related to the absolute magnitude or stellar mass (Best et al. 2007; Mittal et al. 2009; Kale et al. 2015). We will check it together with cluster richness in Sect. 3.4, and here show the probability distribution of two power-limited complete samples of radio BCGs along the absolute magnitude in the left panel of Figure 6. The KS test tells that the BCG samples of $24.6<\log P_{1.4 \mathrm{GHz}}<25.0$ and of $\log P_{1.4 \mathrm{GHz}}>25.0$ have different distributions of the absolute magnitude or stellar mass with an obvious shift of more powerful radio BCGs to optical brighter magnitude, which echos with the previous conclusion (e.g., Best et al. 2007; von der Linden et al. 2007; Antognini et al. 2012; Hogan et al. 2015) that the radio loud fraction is larger for more massive BCGs for a given flux density threshold.

\subsection{Radio power of BCGs versus cluster properties}

We investigate here the possible correlation between the radio power of BCGs and cluster environments. Most of previous studies have worked on the X-ray cluster samples (e.g. Lin \& Mohr 2007; Ma et al. 2013; Hogan et al. 2015) and check if the BCG radio emission or the fraction of radio BCGs depends on cluster dynamical state or cluster mass. Observations of small sample of clusters show that BCGs in relax clusters have higher possibility to be radio loud than those in unrelaxed clusters and that radio power of BCGs seems to correlate with dynamical parameters of clusters (e.g. Peres et al. 1998; Mittal et al. 2009; Kale et al. 2015). Here we work on the optical cluster sample.

Two classes of cluster parameters are investigated for their relation with BCG radio emission power. First is on cluster mass. Mass of galaxy clusters is in general estimated from the X-ray data (e.g. Reiprich \& Böhringer 2002). Up to now only a few thousand galaxy clusters have their mass so estimated (e.g. Vikhlinin et al. 2009; Mantz et al. 2010; Piffaretti et al. 2011). We get X-ray estimated mass for 198 host clusters with radio BCGs from the compiled catalog by Wen \& Han (2015b) and the SZ-estimated masses for 90 clusters from the recent Planck cluster catalog (Planck Collaboration et al. 2015). The cluster richness $R_{L *}$ derived from the total optical luminosity of member galaxies is used as an optical mass proxy of clusters as verified by Wen \& Han (2015b).

In the middle-rank of Figure 5, the BCG radio power is plotted against $R_{L *}$ and the cluster mass estimated from X-ray and SZ observations. Data scattered but show weak correlations in all three panels which indicates the tendency that more powerful radio BCGs are hosted by more massive clusters. The BCGs in clusters with smaller richnesses or less masses have a distribution of radio power peaked at a smaller power than those in richer clusters, which is consistent the results of Lin \& Mohr (2007) and Ma et al. (2013). This is understandable, because BCGs are brighter in richer clusters (Wen et al. 2012) which could produce a bit stronger radio emission (see discussion above). It is intriguing, however, to see no correlation between the radio power and cluster richness for the radio BCGs of a complete sample of massive clusters (the left panel of middle-rank of Figure 5), and no difference is found for the cluster richness distributions of two radio power-limited complete subsamples of BCGs (see the middle panel of Figure 6). Such a result implies for no dependence of BCG radio emission on cluster richness (Antognini et al. 2012). Noticed that these two complete samples of BCGs are hosted by clusters with only a small range of richness and that the correlation is shown between radio power and $\mathrm{M}_{\mathrm{SZ}}$. Therefore whether there is any dependence of $\mathrm{BCG}$ radio power on richness or cluster mass has to be concluded by further investigations of a large cluster sample with a much large range of mass or richness.

Next is on dynamical states of galaxy clusters. In principle the three-dimensional mass and velocity distributions of gas and member galaxies should be measured to describe if a galaxy cluster is dynamically relaxed or in a state of merging or dynamically disturbed. In practice, one-dimensional measurements of velocity dis- 

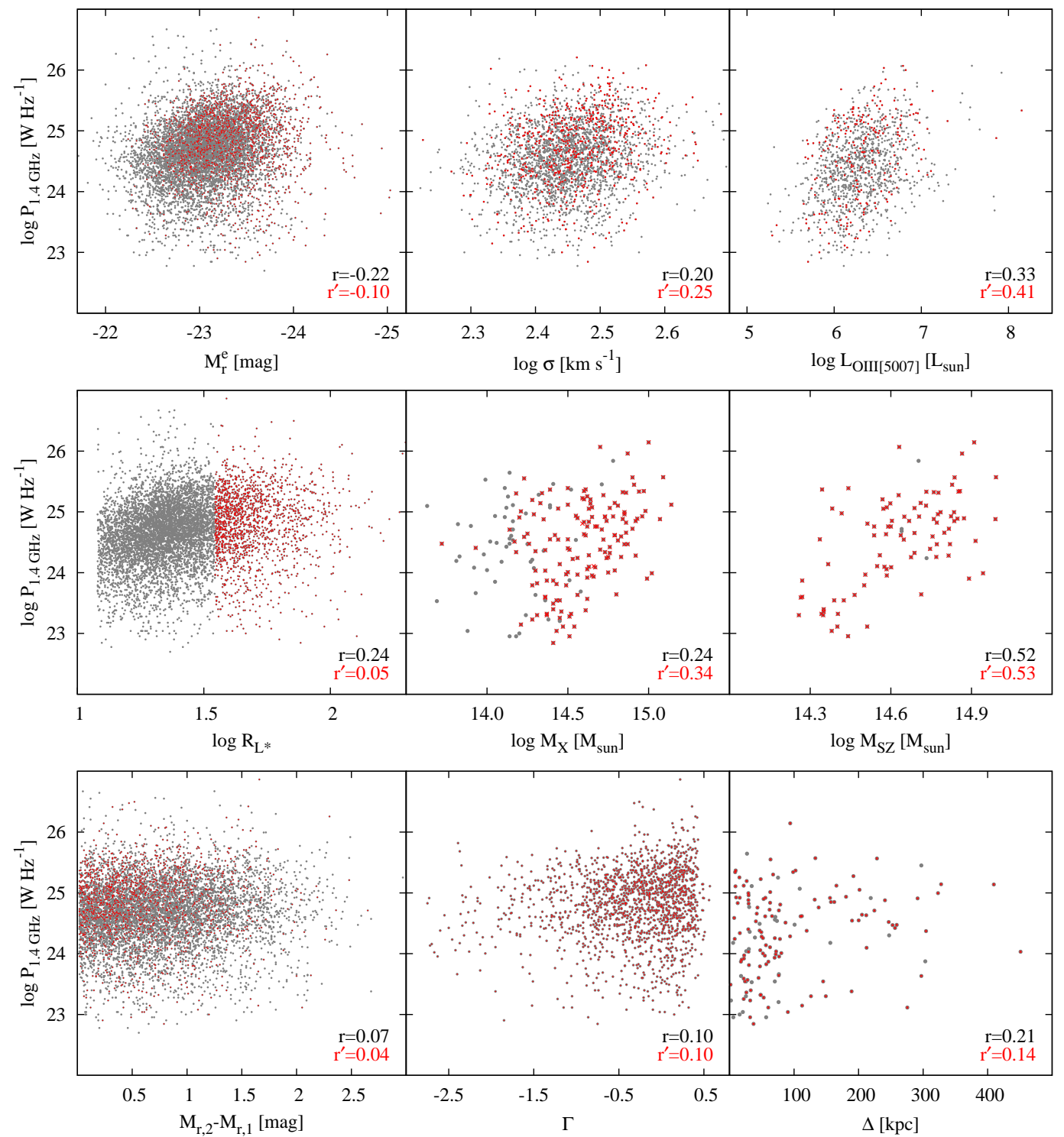

Figure 5. Radio power of BCGs are plotted against BCG optical parameters in top panels (left for the evolution corrected $r$-band absolute magnitude $\mathrm{M}_{\mathrm{r}}^{\mathrm{e}}$ , middle for stellar velocity dispersion $\sigma$, and right for the luminosity of [O III] lines $\left.\mathrm{L}_{[\mathrm{O}} \mathrm{III}\right]$ ), cluster properties in middle rank panels (left for richness $R_{L *}$, middle for $\mathrm{X}$-ray estimated mass $\mathrm{M}_{\mathrm{X}}$ and right for $\mathrm{SZ}$-estimated mass $\mathrm{M}_{\mathrm{SZ}}$ ) and cluster dynamic states in bottom panels (left for BCG dominance $\mathrm{M}_{\mathrm{r}, 2}-\mathrm{M}_{\mathrm{r}, 1}$, middle for optical dynamical parameter $\Gamma$ from Wen \& Han (2013), and right for the offset $\Delta$ of X-ray peak from BCG), if these parameters are available (see text). The radio BCGs in a complete sample of massive clusters of $M_{500}>2 \times 10^{14} \mathrm{M}_{\odot}$ are indicated by (little red) crosses. The Spearman correlation coefficients are given in the bottom-right corner of each panel for both all radio BCGs $(r)$ and the radio-flux-limited complete sample $\left(r^{\prime}\right)$.

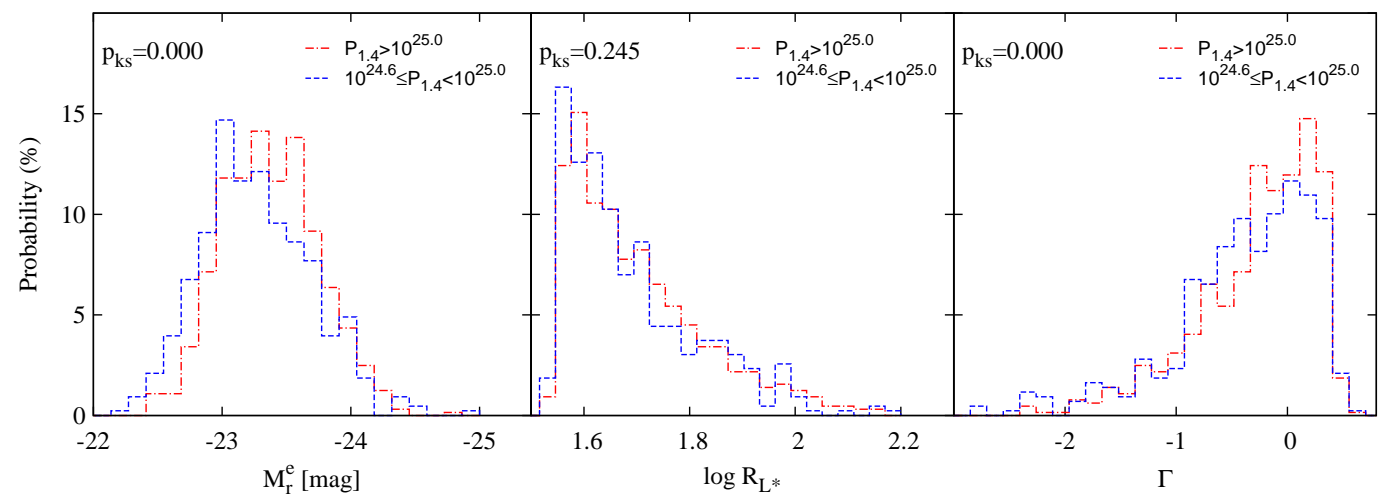

Figure 6. The probability distributions for two power-limited complete sub-samples of a massive cluster sample of $M_{500}>2 \times 10^{14} \mathrm{M}_{\odot}$ along the evolution corrected $r$-band absolute magnitude $\mathrm{M}_{\mathrm{r}}^{\mathrm{e}}\left(\right.$ left), cluster richness $R_{L *}$ (middle) and the cluster dynamic state parameter $\Gamma$ (right). The KS-test probability for the two subsamples is given in the upper-left corner of each panel. 

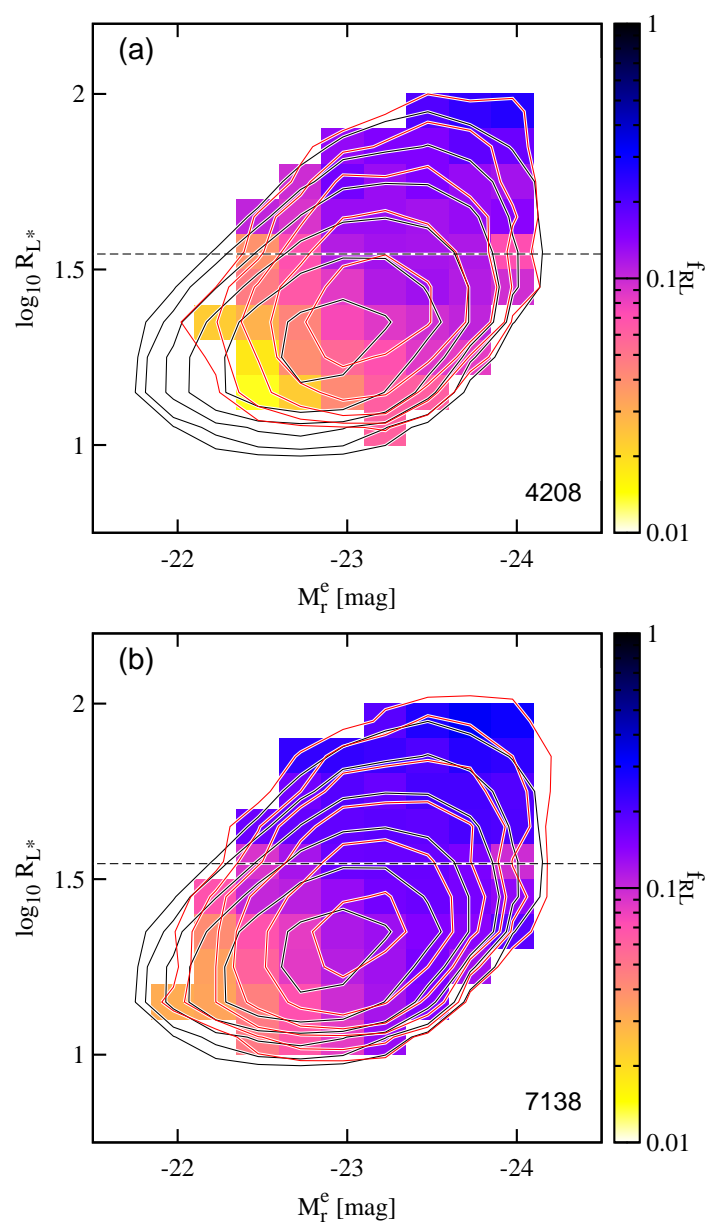

Figure 7. The fraction of radio loud BCGs, $f_{\mathrm{RL}}$, varies with the $r$-band absolute magnitude of $\mathrm{BCG}$ and cluster richness. The upper panel is calculated by using 4,208 radio BCGs with a power-limited threshold of $P_{1.4 \mathrm{GHz}}>10^{24.6} \mathrm{~W} \mathrm{~Hz}^{-1}$, and the lower panel by using 7,138 radio BCGs with a flux limit of $S_{1.4 \mathrm{GHz}}>5 \mathrm{mJy}$, both of which are compared with 62,686 BCGs in the optical parent cluster sample. Above the dash line of $R_{L *}=35$ is a complete BCG sample of massive clusters of $z<0.45$. A pixel is shown only if there have more than 10 radio BCGs. Overlaid are red contours for the number distribution of radio BCGs at levels of $5 \times 2^{n}$ (n $=0,1,2,3,4,5)$ per pixel, and black contours for 62,686 BCGs of the parent sample at levels of $5 \times 2^{n} \times D(\mathrm{n}=0,1,2,3,4,5)$, and $D=62,686 / 4,208$ or $62,686 / 7,138$ for the two samples, respectively.

tribution of member galaxies (e.g. Dressler \& Shectman 1988) and the two-dimensional distribution of hot gas (e.g. Mann \& Ebeling 2012) or member galaxies (e.g. Wen \& Han 2013) have been quantitatively analyzed for cluster dynamical states. We here get the optical dynamical parameters $\Gamma$ of 1,594 clusters from Wen \& Han (2013) and from new calculations and the offsets of BCGs from the peak of X-ray images for 149 host clusters from Piffaretti et al. (2011). We noticed that the BCG dominance $M_{r, 2}-M_{r, 1}$ can statistically be used as being an indicator of dynamical parameter for galaxy clusters (see Wen \& Han 2013). These dynamical parameters are not significant correlated with radio power of BCGs, as shown in the lower panels of Figure 5. However, the distributions of optical dynamical parameters $\Gamma$ are slightly different for the two radio-power-limited complete samples of BCGs in massive clusters, as shown in the right panel of Figure 6. Slightly more radiopowerful BCGs are detected in more dynamically relaxed clusters, which is consistent with the results of Kale et al. (2015).

\subsection{Fraction of radio loud BCGs versus BCG magnitude and cluster richness}

Because of large data scatter, it is very hard to correlate the radio power with BCG properties and cluster environments as shown above. The fraction of radio loudness, $f_{\mathrm{RL}}$, defined as the percentage or the ratio between the radio detected objects and the full sample, has been used to check the possible dependence of radio emission on other galaxy properties (e.g. Best et al. 2005b). The fraction of radio BCGs has been related to either the absolute magnitude of BCGs (e.g. von der Linden et al. 2007; Best et al. 2007; Croft et al. 2007; Antognini et al. 2012) or the X-ray luminosity and richness of galaxy clusters (e.g., Ma et al. 2013), but not both yet. Here we take this large sample of radio BCGs to study the dependence of radio laud fraction in two-dimensions on both BCG and cluster properties.

Remember that 7,138 radio loud BCGs were identified from a parent sample of 62,686 optical clusters by using the NVSS and FIRST survey data with a total flux density threshold of $S_{1.4 \mathrm{GHz}}>$ $5 \mathrm{mJy}$, and 4,208 of which are above the radio power threshold of $P_{1.4 \mathrm{GHz}}=10^{24.6} \mathrm{~W} \mathrm{~Hz}^{-1}$ in the redshift range $0.05<z \leq 0.45$ (see Figure 3). We define the radio loud fraction $f_{\mathrm{RL}}\left(\mathrm{M}_{\mathrm{r}}^{\mathrm{e}}, \mathrm{R}_{\mathrm{L} *}\right)$ as the number ratio of these radio BCGs to the BCG numbers of the parent cluster sample for a given small range of the BCG magnitude and cluster richness. The radio fraction is then checked in two-dimensions for its possible dependence on the BCG luminosity and cluster richness. As shown in Figure 7, for both the powerlimited radio BCG sample or the flux-limited radio BCG sample, there is a very clear tendency that the radio fraction increases with both BCG magnitude and cluster richness, from $\sim 1 \%$ in the lowerleft corner to $\sim 20 \%$ in the top-right corner that is for very bright BCGs in massive rich clusters. To disentangle their effects, the dependence of radio fraction on one parameter should be checked in Figure 7 only in a small range of the other parameter. Integrating data in Figure 7 over one dimension give a global dependence of the radio fraction on the other dimension, echoing the results previously obtained by Croft et al. (2007); von der Linden et al. (2007); Best et al. (2007); Antognini et al. (2012); Ma et al. (2013).

We conclude that the BCG mass and cluster environments only statistically affect the BCG radio emission, but not through individual cases.

\section{RADIO LUMINOSITY FUNCTION OF BCGS}

Noticed that the fraction of radio BCGs depends on the threshold of radio observation. The radio power data are very scattered against BCG and cluster properties. Luminosity function is an important tool to study the evolution of space population. We now work on radio luminosity function of BCGs, and check the dependence of the function on the BCG and cluster properties.

The luminosity function $\Phi(P)$ stands for the comoving space density of a kind of objects in a complete sample for a given luminosity $P$ (e.g. Auriemma et al. 1977; Condon 1989). Considering the possible cosmological evolution, the global average space density should be calculated at the present epoch for the local luminosity function (e.g. Condon 1989; Mauch \& Sadler 2007). As shown in section 2, we have got a complete sample of massive clusters of $M_{500}>2 \times 10^{14} \mathrm{M}_{\odot}$ (i.e. $R_{\mathrm{L} *}>35$ ) within the redshift range of $0.05<z \leq 0.45$, from which 1,594 BCGs have been detected in radio surveys above the flux-limit of $S_{1.4 \mathrm{GHz}}>5 \mathrm{mJy}$ (see the crosses in Figure 3). This forms a complete radio BCG sample for radio luminosity function of BCGs. 

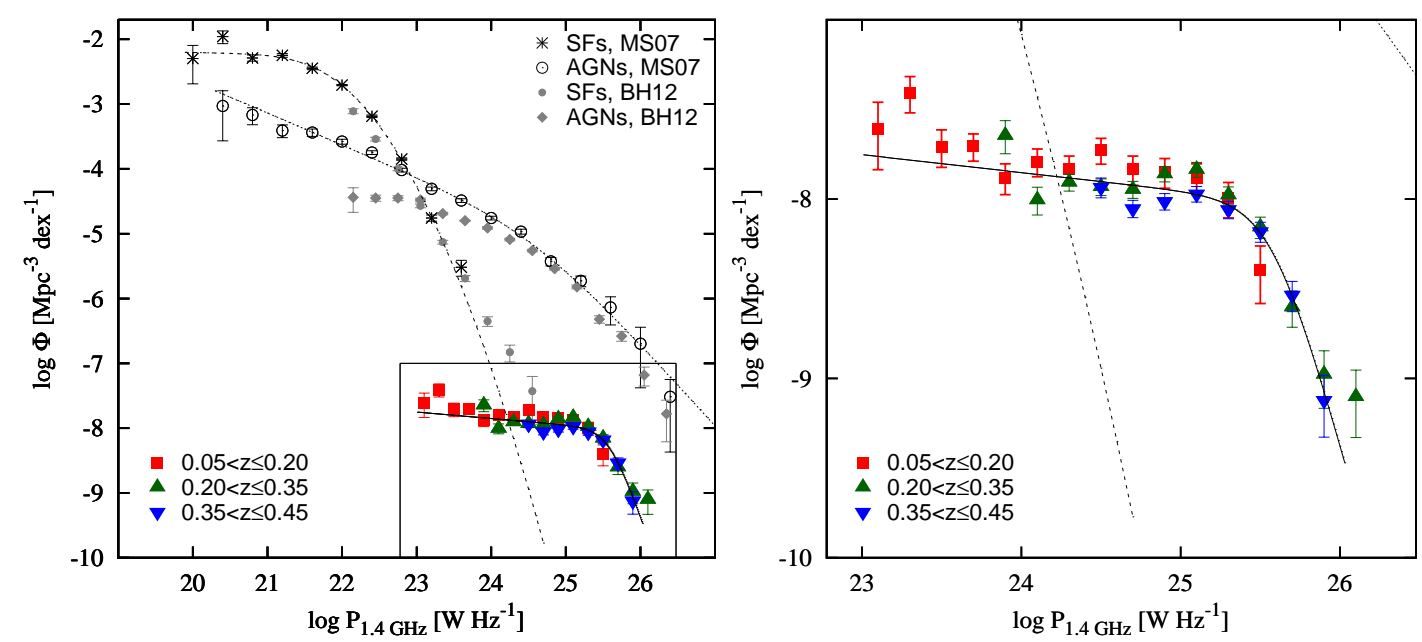

Figure 8. Radio luminosity functions of BCGs derived for subsamples in three redshift ranges, compared with radio luminosity functions of star-forming galaxies and AGNs obtained by Mauch \& Sadler (2007) and Best \& Heckman (2012). The solid line is the best fitting to radio luminosity functions of all BCGs, and dash and dotted lines stand for the fitting to the function for AGNs and star-forming in Mauch \& Sadler (2007). An enlarged part for radio luminosity functions of BCGs is shown in the right panel.

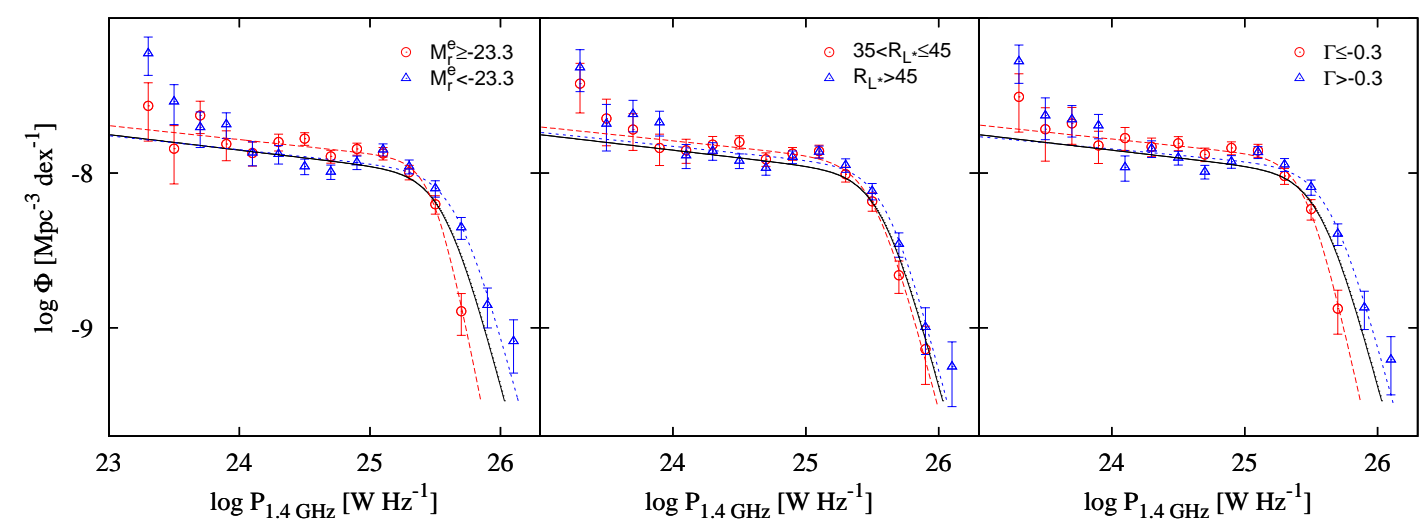

Figure 9. Radio luminosity functions of BCGs in two subsamples with different ranges of BCG luminosity (left), cluster richness (middle) and dynamic states (right). The black solid lines is the best fitting for all radio BCGs, the same as that in Figure 8, and dash and dot lines are best fittings for two subsamples. For better comparison, the radio luminosity functions of BCGs in two subsamples have been re-scaled to that of the full sample of BCGs according to source numbers.

The radio luminosity functions $\Phi(P)$ are calculated in the standard way, as $\Phi(P)=\sum_{i} 1 / V_{i}$. Here $V_{i}$ is the volume in which the $i$ th $\mathrm{BCG}$ with a radio power between $P$ to $P+d P$ could be detected (Schmidt 1968; Condon 1989; Best \& Heckman 2012). For $N$ sources of a complete sample detected in the redshift range of $z_{\min }<z_{i}<z_{\max }$ in a given sky region, all of them have $V_{i}=V_{z_{\max }}-V_{z_{\min }}$, so that $\Phi(P)=\sum_{i=1}^{N} 1 / V_{i}=$ $N /\left(V_{z_{\max }}-V_{z_{\min }}\right)$. Note here that the sky area of 9,376 square degree (i.e $2.85 \mathrm{sr}$ ) for the the complete sample of massive clusters and hence the radio BCGs has to taken into account for calculation of $V_{i}$. To study if the radio luminosity functions of BCGs evolve with redshift, we divide the complete sample into three sub-samples with redshift ranges of $0.05<z \leq 0.20,0.20<z \leq 0.35$, and $0.35<z \leq 0.45$. Their radio luminosity functions are listed in Table 4 and plotted in Figure 8. The uncertainties of $\Phi(P)$ here include only the statistical Poissonian errors, and hence are underestimated for some bins with small number of objects.

As shown clearly in Figure 8, no evolution with redshift can be found from radio luminosity functions obtained from the subsamples of three different redshift ranges, which is consistent with previous results for radio galaxies (e.g., Sadler et al.
2007; Donoso et al. 2009; McAlpine \& Jarvis 2011; Simpson et al. 2012). Following Mauch \& Sadler (2007), we fit the radio luminosity functions of three subsamples together with a two power-law analogous:

$\Phi\left(P_{1.4 \mathrm{GHz}}\right)=\frac{C_{0}}{\left(P_{1.4 \mathrm{GHz}} / P_{0}\right)^{\alpha}+\left(P_{1.4 \mathrm{GHz}} / P_{0}\right)^{\beta}}$,

and obtained the best fitted parameters as

$$
\begin{aligned}
C_{0} & =(9.4 \pm 1.4) \times 10^{-9} \mathrm{Mpc}^{-3} \mathrm{dex}^{-1} ; \\
P_{0} & =(39.9 \pm 5.5) \times 10^{24} \mathrm{~W} \mathrm{~Hz}^{-1} ; \\
\alpha & =3.43 \pm 0.79 ; \\
\beta & =0.12 \pm 0.06 .
\end{aligned}
$$

Comparing to the radio luminosity functions of star forming galaxies and AGNs obtained by Mauch \& Sadler (2007) and Best \& Heckman (2012), we find that the space density of radio BCGs is significantly lower than AGNs when $P_{1.4 \mathrm{GHz}} \gtrsim$ $10^{24.5} \mathrm{~W} \mathrm{~Hz}{ }^{-1}$ or lower than star-forming galaxies when $P_{1.4 \mathrm{GHz}} \leq 10^{24.5} \mathrm{~W} \mathrm{~Hz}^{-1}$. The slope and the tuning points are 
Table 4. Radio luminosity functions at $1.4 \mathrm{GHz}$ for radio loud BCGs in three redshift ranges (only for more than 5 objects each bin).

\begin{tabular}{|c|c|c|c|c|c|c|}
\hline \multirow[b]{2}{*}{$\begin{array}{c}\log P_{1.4 \mathrm{GHz}} \\
\mathrm{W} \mathrm{Hz}-1\end{array}$} & \multicolumn{2}{|c|}{$0.05<z \leq 0.20$} & \multicolumn{2}{|c|}{$0.20<z \leq 0.35$} & \multicolumn{2}{|c|}{$0.35<z \leq 0.45$} \\
\hline & $N$ & $\begin{array}{c}\log \Phi \\
\mathrm{Mpc}^{-3} \mathrm{dex}^{-1}\end{array}$ & $N$ & $\begin{array}{c}\log \Phi \\
\mathrm{Mpc}^{-3} \mathrm{dex}^{-1}\end{array}$ & $N$ & $\begin{array}{c}\log \Phi \\
\mathrm{Mpc}^{-3} \mathrm{dex}^{-1}\end{array}$ \\
\hline 23.1 & 10 & $-7.39_{-0.17}^{+0.12}$ & & & & \\
\hline 23.3 & 22 & $-7.34_{-0.10}^{+0.08}$ & & & & \\
\hline 23.5 & 22 & $-7.62_{-0.10}^{+0.08}$ & & & & \\
\hline 23.7 & 39 & $-7.63_{-0.08}^{+0.07}$ & & & & \\
\hline 23.9 & 28 & $-7.85_{-0.09}^{+0.08}$ & 21 & $-7.69_{-0.11}^{+0.09}$ & & \\
\hline 24.1 & 33 & $-7.78_{-0.08}^{+0.07}$ & 34 & $-7.98_{-0.08}^{+0.07}$ & & \\
\hline 24.3 & 26 & $-7.88_{-0.10}^{+0.08}$ & 92 & $-7.88_{-0.05}^{+0.04}$ & & \\
\hline 24.5 & 38 & $-7.72_{-0.08}^{+0.07}$ & 86 & $-7.95_{-0.05}^{+0.04}$ & 61 & $-7.95_{-0.06}^{+0.05}$ \\
\hline 24.7 & 31 & $-7.81_{-0.09}^{+0.07}$ & 93 & $-7.91_{-0.05}^{+0.04}$ & 77 & $-8.08_{-0.05}^{+0.05}$ \\
\hline 24.9 & 32 & $-7.79_{-0.08}^{+0.07}$ & 105 & $-7.86_{-0.04}^{+0.04}$ & 92 & $-8.00_{-0.05}^{+0.04}$ \\
\hline 25.1 & 28 & $-7.85_{-0.10}^{+0.08}$ & 117 & $-7.81_{-0.04}^{+0.04}$ & 100 & $-7.97_{-0.05}^{+0.04}$ \\
\hline 25.3 & 21 & $-7.97_{-0.11}^{+0.09}$ & 87 & $-7.94_{-0.05}^{+0.04}$ & 82 & $-8.05_{-0.05}^{+0.05}$ \\
\hline 25.5 & 7 & $-8.45_{-0.21}^{+0.14}$ & 55 & $-8.14_{-0.06}^{+0.06}$ & 60 & $-8.19_{-0.06}^{+0.05}$ \\
\hline 25.7 & 7 & $-8.45_{-0.21}^{+0.14}$ & 21 & $-8.56_{-0.11}^{+0.09}$ & 27 & $-8.54_{-0.09}^{+0.08}$ \\
\hline 25.9 & & & 8 & $-8.98_{-0.19}^{+0.13}$ & 6 & $-9.19_{-0.23}^{+0.15}$ \\
\hline 26.1 & & & 6 & $-9.10_{-0.23}^{+0.15}$ & & \\
\hline Total & 348 & & 727 & & 519 & \\
\hline
\end{tabular}

Table 5. Fitting parameters of radio luminosity functions of BCG samples.

\begin{tabular}{lcrcc}
\hline \hline BCG Sample & \multicolumn{1}{c}{$C_{0}$} & \multicolumn{1}{c}{$P_{0}$} & $\alpha$ & $\beta$ \\
\hline All & $9.4 \pm 1.4$ & $39.9 \pm 5.5$ & $3.43 \pm 0.79$ & $0.12 \pm 0.06$ \\
$M_{\mathrm{r}}^{\mathrm{e}} \geq-23.3$ & $6.3 \pm 0.6$ & $32.4 \pm 2.4$ & $4.46 \pm 0.81$ & $0.08 \pm 0.04$ \\
$M_{\mathrm{r}}^{\mathrm{e}}<-23.3$ & $4.5 \pm 1.1$ & $50.9 \pm 11.1$ & $3.37 \pm 1.24$ & $0.14 \pm 0.08$ \\
$35<R_{\mathrm{L} *} \leq 45$ & $5.8 \pm 0.8$ & $33.6 \pm 3.8$ & $3.59 \pm 0.67$ & $0.09 \pm 0.05$ \\
$R_{\mathrm{L} *}>45$ & $5.1 \pm 1.0$ & $44.9 \pm 7.8$ & $3.48 \pm 0.97$ & $0.13 \pm 0.07$ \\
$\Gamma \leq-0.3$ & $5.5 \pm 0.7$ & $30.5 \pm 3.1$ & $3.75 \pm 0.77$ & $0.08 \pm 0.05$ \\
$\Gamma>-0.3$ & $5.6 \pm 1.1$ & $47.2 \pm 8.4$ & $3.40 \pm 1.00$ & $0.12 \pm 0.07$ \\
\hline
\end{tabular}

Notes: $C_{0}$ in $10^{-9} \mathrm{Mpc}^{-3} \mathrm{dex}^{-1}, P_{0}$ in $10^{24} \mathrm{~W} \mathrm{~Hz}^{-1}$.

very different from those of radio luminosity functions of AGNs and star-forming galaxies.

To check the dependence of radio luminosity functions on the BCG and cluster properties, we divide the 1,594 radio BCGs into two half sub-samples according to the BCG absolute magnitude, cluster richness and also dynamical parameter of clusters. The radio luminosity function of every two subsamples are shown in Figure 9 and the best fitting parameters are listed in Table 5. The functions are normalized according to BCG numbers for easy comparison. We do see different radio luminosity functions for BCGs of different ranges of absolute magnitude or different dynamical states. However, no significant difference can be found for the two BCG samples of slightly different richness ranges. The results imply that more radio power BCGs are associated with optically bright BCGs in the relaxed clusters, confirming the result in Section 3.4 and conclusions by Mittal et al. (2009) and Kale et al. (2015).

\section{SUMMARY AND CONCLUSIONS}

Basing on the largest optical catalog of galaxy clusters of WH15 and the largest radio survey database of the NVSS and FIRST, we identified a large sample of 7,138 radio loud BCGs.

We found that radio power data of BCGs are rather scattered when they are plotted against the BCG absolute magnitudes and cluster mass proxies or cluster dynamical parameters. Very weak or no significant correlations can be found between the radio power and the BCG or cluster parameters. The fraction of radio laud BCGs has been checked against the BCG absolute magnitudes and cluster richness, and we confirm that radio loud fraction of BCGs does increase with BCG luminosity and cluster richness in twodimension. By using the large complete BCG sample, we construct the radio luminosity functions of BCGs, and do not find any redshift evolution in the redshift range of $0.05<z \leq 0.45$. Radio luminosity functions are different for BCGs with different ranges of BCG luminosity and the cluster dynamical parameter. We conclude that BCGs are more probably radio powerful if they have a larger absolute magnitude and resident in more relaxed cluster.

\section{ACKNOWLEDGMENTS}

The authors are supported by the National Natural Science Foundation (No. 11473034) and by the Strategic Priority Research Program "The Emergence of Cosmological Structures" of the Chinese Academy of Sciences, Grant No. XDB09010200. Funding for SDSS-III has been provided by the Alfred P. Sloan Foundation, the Participating Institutions, the National Science Foundation, and the US Department of Energy. The SDSS-III Web site is http://www.sdss3.org/. 


\section{REFERENCES}

Aihara H., et al., 2011, ApJS, 193, 29

Alam S., et al., 2015, ApJS, 219, 12

Allen S. W., Evrard A. E., Mantz A. B., 2011, ARA\&A, 49, 409

Antognini J., Bird J., Martini P., 2012, ApJ, 756, 116

Auriemma C., Perola G. C., Ekers R. D., Fanti R., Lari C., Jaffe W. J., Ulrich M. H., 1977, A\&A, 57, 41

Baldwin J. A., Phillips M. M., Terlevich R., 1981, PASP, 93, 5

Ball R., Burns J. O., Loken C., 1993, AJ, 105, 53

Becker R. H., White R. L., Helfand D. J., 1995, ApJ, 450, 559

Best P. N., Heckman T. M., 2012, MNRAS, 421, 1569

Best P. N., Kauffmann G., Heckman T. M., Ivezić Ž., 2005a, MNRAS, 362,9

Best P. N., Kauffmann G., Heckman T. M., Brinchmann J., Charlot S., Ivezić Ž., White S. D. M., 2005b, MNRAS, 362, 25

Best P. N., von der Linden A., Kauffmann G., Heckman T. M., Kaiser C. R., 2007, MNRAS, 379, 894

Best P. N., Ker L. M., Simpson C., Rigby E. E., Sabater J., 2014, MNRAS, 445,955

Brinchmann J., Charlot S., White S. D. M., Tremonti C., Kauffmann G., Heckman T., Brinkmann J., 2004, MNRAS, 351, 1151

Burns J. O., 1990, AJ, 99, 14

Burns J. O., White R. A., Hough D. H., 1981, AJ, 86, 1

Calvani M., Fasano G., Franceschini A., 1989, AJ, 97, 1319

Condon J. J., 1989, ApJ, 338, 13

Condon J. J., Cotton W. D., Greisen E. W., Yin Q. F., Perley R. A., Taylor G. B., Broderick J. J., 1998, AJ, 115, 1693

Croft S., de Vries W., Becker R. H., 2007, ApJ, 667, L13

Donoso E., Best P. N., Kauffmann G., 2009, MNRAS, 392, 617

Dressler A., Shectman S. A., 1988, AJ, 95, 985

Fabian A. C., 1994, ARA\&A, 32, 277

Hogan M. T., et al., 2015, MNRAS, 453, 1201

Kale R., Venturi T., Cassano R., Giacintucci S., Bardelli S., Dallacasa D., Zucca E., 2015, A\&A, 581, A23

Kauffmann G., et al., 2003, MNRAS, 346, 1055

Kewley L. J., Dopita M. A., Sutherland R. S., Heisler C. A., Trevena J., 2001, ApJ, 556, 121

Koester B. P., et al., 2007, ApJ, 660, 239

Lin Y.-T., Mohr J. J., 2007, ApJS, 170, 71

Liu F., Wen Z., Han J., Meng X., 2012a, Science China Physics, Mechanics, and Astronomy, 55, 354

Liu F. S., Mao S., Meng X. M., 2012b, MNRAS, 423, 422

Ma C.-J., McNamara B. R., Nulsen P. E. J., Schaffer R., Vikhlinin A., 2011, ApJ, 740, 51

Ma C.-J., McNamara B. R., Nulsen P. E. J., 2013, ApJ, 763, 63

Machalski J., Godlowski W., 2000, A\&A, 360, 463

Maiolino R., Rieke G. H., 1995, ApJ, 454, 95

Mann A. W., Ebeling H., 2012, MNRAS, 420, 2120

Mantz A., Allen S. W., Ebeling H., Rapetti D., Drlica-Wagner A., 2010, MNRAS, 406, 1773

Mao M. Y., et al., 2012, MNRAS, 426, 3334

Marconi A., Hunt L. K., 2003, ApJ, 589, L21

Mauch T., Sadler E. M., 2007, MNRAS, 375, 931

McAlpine K., Jarvis M. J., 2011, MNRAS, 413, 1054

McAlpine K., Jarvis M. J., Bonfield D. G., 2013, MNRAS, 436, 1084

McCarthy P. J., 1993, ARA\&A, 31, 639

McNamara B. R., Nulsen P. E. J., 2007, ARA\&A, 45, 117

Miller C. J., et al., 2005, AJ, 130, 968

Mittal R., Hudson D. S., Reiprich T. H., Clarke T., 2009, A\&A, 501, 835

O'Dea K. P., et al., 2010, ApJ, 719, 1619

Padovani P., Miller N., Kellermann K. I., Mainieri V., Rosati P., Tozzi P., 2011, ApJ, 740, 20

Padovani P., Bonzini M., Kellermann K. I., Miller N., Mainieri V., Tozzi P., 2015, MNRAS, 452, 1263

Peres C. B., Fabian A. C., Edge A. C., Allen S. W., Johnstone R. M., White D. A., 1998, MNRAS, 298,416
Piffaretti R., Arnaud M., Pratt G. W., Pointecouteau E., Melin J.-B., 2011, A\&A, 534, A109

Planck Collaboration et al., 2015, preprint, (arXiv:1502.01598)

Prescott M., et al., 2016, preprint, (arXiv:1601.00003)

Rafferty D. A., McNamara B. R., Nulsen P. E. J., Wise M. W., 2006, ApJ, 652, 216

Reiprich T. H., Böhringer H., 2002, ApJ, 567, 716

Sadler E. M., et al., 2002, MNRAS, 329, 227

Sadler E. M., et al., 2007, MNRAS, 381, 211

Saunders R., Baldwin J. E., Rawlings S., Warner P. J., Miller L., 1989, MNRAS, 238, 777

Schmidt M., 1968, ApJ, 151, 393

Sikora M., Stasińska G., Koziel-Wierzbowska D., Madejski G. M., Asari N. V., 2013, ApJ, 765, 62

Simpson C., et al., 2012, MNRAS, 421, 3060

Stott J. P., et al., 2012, MNRAS, 422, 2213

Tremaine S., et al., 2002, ApJ, 574, 740

Tremonti C. A., et al., 2004, ApJ, 613, 898

Vikhlinin A., et al., 2009, ApJ, 692, 1033

Wen Z. L., Han J. L., 2013, MNRAS, 436, 275

Wen Z. L., Han J. L., 2015a, MNRAS, 448, 2

Wen Z. L., Han J. L., 2015b, ApJ, 807, 178

Wen Z. L., Han J. L., Liu F. S., 2012, ApJS, 199, 34

Willott C. J., Rawlings S., Blundell K. M., Lacy M., 1999, MNRAS, 309, 1017

Zhao J.-H., Burns J. O., Owen F. N., 1989, AJ, 98, 64

van Velzen S., Falcke H., Schellart P., Nierstenhöfer N., Kampert K.-H., 2012, A\&A, 544, A18

von der Linden A., Best P. N., Kauffmann G., White S. D. M., 2007, MNRAS, 379,867 